\title{
Some Implications of an Alternate Equation for the BCS Energy Gap
}

\author{
${\text { Gulshan Prakash Malik }{ }^{1 *} \text {, Manuel de Llano }}^{2}$ \\ ${ }^{1}$ Theory Group, School of Environmental Sciences, Jawaharlal Nehru University, New Delhi, India \\ ${ }^{2}$ Instituto de Investigaciones en Materiales, Universidad Nacional Autónoma de México Apdo, México City, México \\ Email: gulshanpmalik@yahoo.com,malik@mail.jnu.ac.in,dellano@unam.mx
}

Received January 12, 2013; revised February 15, 2013; accepted March 1, 2013

Copyright (C) 2013 Gulshan Prakash Malik, Manuel de Llano. This is an open access article distributed under the Creative Commons Attribution License, which permits unrestricted use, distribution, and reproduction in any medium, provided the original work is properly cited.

\begin{abstract}
A set of generalized-BCS equations (GBCSEs) was recently derived from a temperature-dependent Bethe-Salpeter equation and shown to deal satisfactorily with the experimental data comprising the $T_{c} s$ and the multiple gaps of a variety of high-temperature superconductors (SCs). These equations are formulated in terms of the binding energies $W_{1}(T), W_{2}(T), \cdots$ of Cooper pairs (CPs) bound via one- and more than one-phonon exchange mechanisms; they contain no direct reference to the gap/s of an SC. Applications of these equations so far were based on the observation that for elemental SCs $\left|W_{01}\right|=\Delta_{0}$ at $T=0$ in the limit of the dimensionless BCS interaction parameter $\lambda \rightarrow 0$. Here $\Delta_{0}$ is the zero-temperature gap whence it follows that the binding energy of a CP bound via one-phonon exchanges at $T=0$ is $2\left|W_{01}\right|$. In this note we carry out a detailed comparison between the GBCSE-based $W_{1}(T)$ and the BCS-based energy gap $\Delta(T)$ for all $0 \leq T \leq T_{c}$ and realistic, non-vanishingly-small values of $\lambda$. Our study is based on the experimental values of $T_{c}$, Debye temperature $\Theta$, and $\Delta_{0}$ of several selected elements including the "bad actors" such as $\mathrm{Pb}$ and $\mathrm{Hg}$. It is thus established that the equation for $W_{1}(T)$ provides a viable alternative to the BCS equation for $\Delta(T)$. This suggests the use of, when required, the equation for $W_{2}(T)$ which refers to CPs bound via two-phonon exchanges, for the larger of the two $T$-dependent gaps of a non-elemental SC. These considerations naturally lead one to the concept of $T$-dependent interaction parameters in the theory of superconductivity. It is pointed out that such a concept is needed both in the well-known approach of Suhl et al. to multi-gap superconductivity and the approach provided by the GBCSEs. Attention is drawn to diverse fields where $T$-dependent Hamiltonians have been fruitfully employed in the past.
\end{abstract}

Keywords: BCS and Generalized-BCS Equations; T-Dependent Equations for BCS Gap and for Binding Energy of Cooper Pairs; Elemental and Composite Superconductors

\section{Introduction}

The BCS equations (BCSEs) [1] for the temperaturedependent energy gap $\Delta(T)$ and the critical temperature $T_{c}$ of a superconductor (SC) are, respectively:

$$
\begin{aligned}
& 1=\lambda \int_{0}^{k_{B} \Theta} \mathrm{d} \xi \frac{\tanh \left[\frac{1}{2 k_{B} T}\left(\xi^{2}+\Delta^{2}\right)^{1 / 2}\right]}{\left(\xi^{2}+\Delta^{2}\right)^{1 / 2}} \\
& 1=\lambda \int_{0}^{\Theta / 2 T_{c}} \mathrm{~d} x \frac{\tanh (x)}{x}
\end{aligned}
$$

"Present address: B-208 Sushant Lok I, GurgaonHaryana, India. where $\lambda \equiv N(0) V$ is the dimensionless BCS interacttion parameter corresponding to a net attractive interaction $V$ that brings about the formation of Cooper pairs (CPs), $N(0)$ being the density of states at the Fermi surface; $\Theta$ the Debye temperature of the $\mathrm{SC}$, and $k_{B}$ the Boltzmann constant.

These equations have recently been generalized [2] to cater to composite superconductors (CSs) by following an approach based on the Bethe-Salpeter equation (BSE). While the BCSEs are based on the one-phonon exchange mechanism for the formation of Cooper pairs, generalized-BCS equations (GBCSEs) invoke the mechanism of 
multi-phonon exchanges, besides the one-phonon exchange mechanism, for the formation of the pairs. Additionally, while BCSEs characterize an elemental SC by a single Debye temperature, GBCSEs characterize a CS by multiple Debye temperatures which take into account its anisotropy.

For a binary CS characterized by two gaps such as $\mathrm{MgB}_{2}$, GBCSEs are [2]:

$$
\begin{aligned}
1= & \lambda_{1}^{c}(T) \int_{\left|W_{1}\right| / 2}^{k_{B} \Theta_{1}^{c}+\left|W_{1}\right| / 2} \mathrm{~d} x \frac{\tanh \left(x / 2 k_{B} T\right)}{x} \\
1= & \lambda_{1}^{c}(T) \int_{\left|W_{2}\right| / 2}^{k_{B} \Theta_{1}^{c}+\left|W_{2}\right| / 2} \mathrm{~d} x \frac{\tanh \left(x / 2 k_{B} T\right)}{x} \\
& +\lambda_{2}^{c}(T) \int_{\left|W_{2}\right| / 2}^{k_{B} \Theta_{2}^{c}+\left|W_{2}\right| / 2} \mathrm{~d} x \frac{\tanh \left(x / 2 k_{B} T\right)}{x} \\
1= & \lambda_{1}^{c}\left(T_{c}\right) \int_{0}^{k_{B} \Theta_{1}^{c}} \mathrm{~d} x \frac{\tanh \left(x / 2 k_{B} T_{c}\right)}{x} \\
& +\lambda_{2}^{c}\left(T_{c}\right) \int_{0}^{k_{B} \Theta_{2}^{c}} \mathrm{~d} x \frac{\tanh \left(x / 2 k_{B} T_{c}\right)}{x},
\end{aligned}
$$

where (3) is obtained from Equation (34) in Ref. [2] by a redefinition of the variable of integration and refers to the situation where CPs are bound via one-phonon exchanges; (4) is a generalization of (3) to the situation where CPs are formed via two-phonon exchanges; (5) is obtained from (4) by demanding that $\left|W_{2}\right|=0$ at $T=T_{c}$; $\lambda_{i}^{c}(T)$ is the interaction parameter due to the $i$ th species of ions in the binary, to be distinguished from $\lambda_{i}$, which denotes the interaction parameter of the same species in its free state, a similar distinction applies to $\Theta_{i}^{c}$ and $\Theta_{i}$. Note that we have written $\lambda_{1,2}^{c}$ as $\lambda_{1,2}^{c}(T)$ for the reason that will become apparent via (12) below.

In the above equations, in our earlier work [2-4], $\left|W_{1}\right|$ has been referred to as the binding energy of CPs bound via one-phonon exchanges. Indeed, that is how it was first introduced in the appropriate BSE in [2]. However, it turned out that $\left|W_{1}\right|$ obtained via (3) very nearly equals $\Delta$ obtained via (1). Since we know that the energy required to break up a CP is $2 \Delta$ because of "Pauli blocking" of states between $E_{F}-\Delta$ and $E_{F}+\Delta$, we should refer to $2\left|W_{1}\right|$ as the binding energy of the pairs bound via the one-phonon exchange mechanism. Likewise, the binding energy of pairs bound via two-phonon exchanges is $2\left|W_{2}\right|$. Even though obvious, we note here that since $2\left|W_{1,2}\right|$ also vanish when $\left|W_{1,2}\right|=0$, the equations used in our earlier work (obtained by putting $\left|W_{1,2}\right|=0$ ) are correct.

The motivation for this note is primarily to establish that insofar as elemental SCs are concerned, use of (3) is equivalent to the use of (1) not only at $T=0$ as follows from our earlier work, but for all $T \leq T_{c}$. Such a demonstration is deemed desirable prior to suggesting the use of (4) whenever a detailed variation of the larger of two gaps of a CS with $T$ is required. This is so because the equivalent of (4) via the variational approach of BCS is not yet available. In Section 2, this exercise is interestingly found to shed light on an alleged universal relation of BCS theory, viz., $2 \Delta_{0} / k T_{c}=3.53$. Additionally, it brings into focus the rather unfamiliar feature of $T$-dependence of $\lambda$ in the theory of superconductivity. This feature is briefly discussed further in Section 3 in the context of multi-gap superconductivity. We recall that multi-gap superconductivity is usually qualitatively addressed in the BCS theory via the seminal approach of Suhl, Matthias and Walker [5]. This approach is juxtaposed with the approach provided by the framework of GBCSEs. Finally, Section 4 sums up our study.

\section{A Comparative Study of BCSE (1) and GBCSE (3)}

1) We note that BCSE (2) for $T_{c}$ follows not only from (1) by demanding that $\Delta=0$ at $T=T_{c}$, but also from (3) by demanding that $\left|W_{1}\right|=0$. This already suggests a connection between $\left|W_{1}\right|$ and $\Delta$.

2) It is readily seen that when $T=0(\tanh =1),(1)$ and (3) yield, respectively:

$$
\Delta_{0}=\frac{k_{B} \Theta}{\sinh (1 / \lambda)}
$$

and

$$
\left|W_{01}\right|=\frac{2 k_{B} \Theta}{\exp (1 / \lambda)-1} .
$$

Hence

$$
\Delta_{0}=\left|W_{01}\right|=2 k_{B} \Theta \exp (-1 / \lambda) \text {, when } \lambda \rightarrow 0 .
$$

Based on this observation, (5) and the equations corresponding to (3) and (4) at $T=0$ were recently used $[3,4]$ to deal with the experimental data related with the $T_{c} s$ and the multiple gaps of a variety of high-temperature SCs (HTSCs). Specifically, it has been shown in these papers that if one identifies $\left|W_{1}\right|$ in (3) with the smaller gap of an HTSC, and $\left|W_{2}\right|$ in (4) with the larger gap, then given any two parameters from the set

$\left\{T_{c}, \Delta_{1}, \Delta_{2}>\Delta_{1}\right\}$ one can calculate the remaining parameter without any arbitrariness. This is similar to what the normal BCS equations achieve for a simple SC.

3) We now propose to go beyond the approximations which led to (8). Specifically, we carry out below a detailed comparison of the implications of (1) and (3) for all $0 \leq T \leq T_{c}$ and realistic values of $\lambda$ (i.e., not $\rightarrow 0$ ). Our study is based on the experimental values of $T_{c}, \Theta$, and $\Delta_{0}$ of several selected elements including the "bad actors" such as $\mathrm{Pb}$ and $\mathrm{Hg}$. 
4) With the input of $T_{c}$ and $\Theta$ into (2), we first determine $\lambda\left(T_{c}\right)$ for the elements listed in Table 1. With $\lambda\left(T_{c}\right)$ known, we calculate $\Delta_{0}$ for these elements via (6), and $\left|W_{01}\right|$ via (7). The results are given in Table 1, along with the experimental values for $\Delta_{0}$ (as well as the gap-to- $T_{c}$ ratio $2 \Delta_{0} / k_{B} T_{c} \equiv R$ ). It is thus seen that: a) generally, $\left|W_{01}\right| \approx \Delta_{0}$; b) Both $\left|W_{01}\right|$ and $\Delta_{0}$ fall short of $\Delta_{0}$ (expt), the mismatch between them being greater for the "bad actors"; c) The values of $2\left|W_{01}\right| / k_{B} T_{c}$ calculated via (3) are closer to the values of $\left[2 \Delta_{0} / k_{B} T_{c}\right]_{\text {expt }}$ than the values of $\left[2 \Delta_{0} / k_{B} T_{c}\right]_{\mathrm{cal}}$ obtained via (1).

5) Using the values of $\lambda\left(T_{c}\right), \Delta_{0}$ and $\left|W_{01}\right|$, we now calculate the dimensionless ratios $\Delta(T) / \Delta_{0}$ and $\left|W_{01}(T)\right| /\left|W_{01}\right|$ for all the elements under consideration, the former via (1) and the latter via (3), for all $0 \leq t \equiv T / T_{c} \leq 1$. These lead to two clusters of curves, plotted in Figure 1, which are found to be more or less similarly compact. The thick dashed-curve between the two clusters in the figure corresponds to a relation obtained via the Gorter-Casimir two-fluid theory of superconductivity, see e.g., Ref. [6]:

$$
n_{s}(t) \equiv N_{s}(t) / N_{s}(0)=1-t^{4},
$$

where $N_{s}$ is the density of superconducting electrons. Inclusion of this curve is suggested by the observation that:

$$
\Delta(T) /\left.\Delta_{0}\right|_{T=0}=W_{01}(T) /\left.W_{01}\right|_{T=0}=n_{s}(0)=1,
$$

and

$$
\Delta(t) /\left.\Delta_{0}\right|_{t=1}=W_{01}(t) /\left.W_{01}\right|_{t=1}=n_{s}(1)=0 .
$$

6) Our considerations so far have been based on the $\lambda$-values that were determined with the input of $T_{c}$ and $\Theta$ of each of the elements. An assertion of the BCS theory is that it has no disposable parameters, which means that the same $\lambda$ must occur in both (1) and (2). Hence one can eliminate $\lambda$ from these equations, which leads to $R=3.53$. We now invert (6) and (7) to obtain

$$
\begin{aligned}
& \lambda\left(\Delta_{0}\right)=\frac{1}{\operatorname{arcsinh}\left(k_{B} \Theta / \Delta_{0}\right)} \\
& \lambda\left(\left|W_{01}\right|\right)=\frac{1}{\ln \left(1+2 k_{B} \Theta /\left|W_{01}\right|\right)} .
\end{aligned}
$$

The values of $\lambda s$ obtained with the input of $\Delta_{0}(\operatorname{expt})\left(=\left|W_{01}\right|\right)$ and $\Theta$ into (10) and (11), respectively, are also given in Table 1. It is thus seen that for each of the listed elements, $\lambda\left(\Delta_{0}\right) \approx \lambda\left(\left|W_{01}\right|\right)$, though the former is always marginally greater than the latter. A significant feature of these $\lambda$-values is that for all the listed elements

$$
\lambda\left(\Delta_{0}\right), \lambda\left(\left|W_{01}\right|\right)>\lambda\left(T_{c}\right) .
$$

Using these values of $\lambda\left(\Delta_{0}\right)$ and $\lambda\left(\left|W_{01}\right|\right)$ in (2) to calculate the values of $T_{c}$ for the elements listed in Table 1, the results are, in degrees Kelvin: $\mathrm{Cd}(0.47,0.47,0.42)$, $\mathrm{Pb}$ (8.7, 8.1, 7.2), $\mathrm{Hg}$ (5.41, 5.14, 4.15), Sn (3.9, 3.83, $3.72)$, In (3.56, 3.46, 3.41), $\mathrm{Tl}(2.6,2.52,2.38), \mathrm{Nb}$ $(10.19,9.88,9.25)$, where the first entry in the parentheses for any element denotes $T_{c}$ corresponding to $\lambda\left(\Delta_{0}\right)$, the second entry to $\lambda\left(\left|W_{01}\right|\right)$, and the third entry denotes the experimental value. It is thus seen that: a) there is a mismatch between the calculated and the experimental values of $T_{c} ; \mathrm{b}$ ) the mismatch is more pronounced for the "bad actors", a circumstance which is responsible for violation of the universality of $R$; and c) The $\boldsymbol{T}_{c} \boldsymbol{s}$ calculated via $\lambda\left(\left|W_{01}\right|\right)$ are invariably closer to the experimental values than those calculated via $\lambda\left(\Delta_{0}\right)$.

7) We believe the inequality in (12) to be important, and while we will discuss it further in the next section in the context of a CS (YBCO) which is characterized by two $\Delta_{0} s$ but one $T_{c}$, let us first delve on its origin. We

Table 1. Experimental values of $T_{c}, \Theta$ and $\Delta_{0}=\left|W_{0}\right|$ are taken from Poole [6]. The $\lambda\left(T_{c}\right)$ is calculated with the input of $T_{c}$ and $\Theta$ into (2), while $\lambda\left(\Delta_{0}\right)$ and $\lambda\left(\left|W_{0}\right|\right)$ are calculated with the input of $\Theta$ and $\Delta_{0}(\operatorname{expt})=\left|W_{0}\right|$ into (10) and (11), respectively. Also, $\Delta_{0}$ and $\left|W_{0}\right|$ are calculated with the input of $\lambda\left(T_{c}\right)$ and $\Theta$ into (7) and (8), respectively.

\begin{tabular}{ccccccccc}
\hline $\mathrm{SC}$ & $T_{c}(K)$ & $\Theta(K)$ & $\left.\Delta_{0}\right|_{\text {cop }}\left|W_{0}\right|(\mathrm{meV}), 2 \Delta_{0} /\left.k_{B} T_{c}\right|_{\text {epp }}$ & $\lambda\left(T_{c}\right)$ & $\lambda\left(\Delta_{0}\right)$ & $\lambda\left(\left|W_{0}\right|\right)$ & $\Delta_{0}(\mathrm{meV}), 2 \Delta_{0} / k_{B} T_{c}$ & $\left|W_{0}\right|(\mathrm{meV}), 2\left|W_{0}\right| / k_{B} T_{c}$ \\
\hline $\mathrm{Cd}$ & 0.42 & 210 & $0.072,3.98$ & 0.1577 & 0.1608 & 0.1607 & $0.064,3.52$ & $0.064,3.53$ \\
$\mathrm{~Pb}$ & 7.2 & 96 & $1.33,4.29$ & 0.3682 & 0.3957 & 0.3849 & $1.099,3.54$ & $1.17,3.78$ \\
$\mathrm{Hg}$ & 4.15 & 88 & $0.824,4.61$ & 0.3145 & 0.3430 & 0.3372 & $0.632,3.53$ & $0.658,3.68$ \\
$\mathrm{Sn}$ & 3.72 & 195 & $0.593,3.7$ & 0.2448 & 0.2477 & 0.2466 & $0.566,3.53$ & $0.575,3.59$ \\
$\mathrm{In}$ & 3.41 & 108 & $0.541,3.68$ & 0.2792 & 0.2826 & 0.2804 & $0.519,3.53$ & $0.533,3.63$ \\
$\mathrm{Tl}$ & 2.38 & 79 & $0.395,3.85$ & 0.2756 & 0.2824 & 0.2802 & $0.362,3.53$ & $0.372,3.62$ \\
$\mathrm{Nb}$ & 9.25 & 276 & $1.55,3.89$ & 0.2840 & 0.2920 & 0.2894 & $1.407,3.53$ & $1.449,3.64$ \\
\hline
\end{tabular}




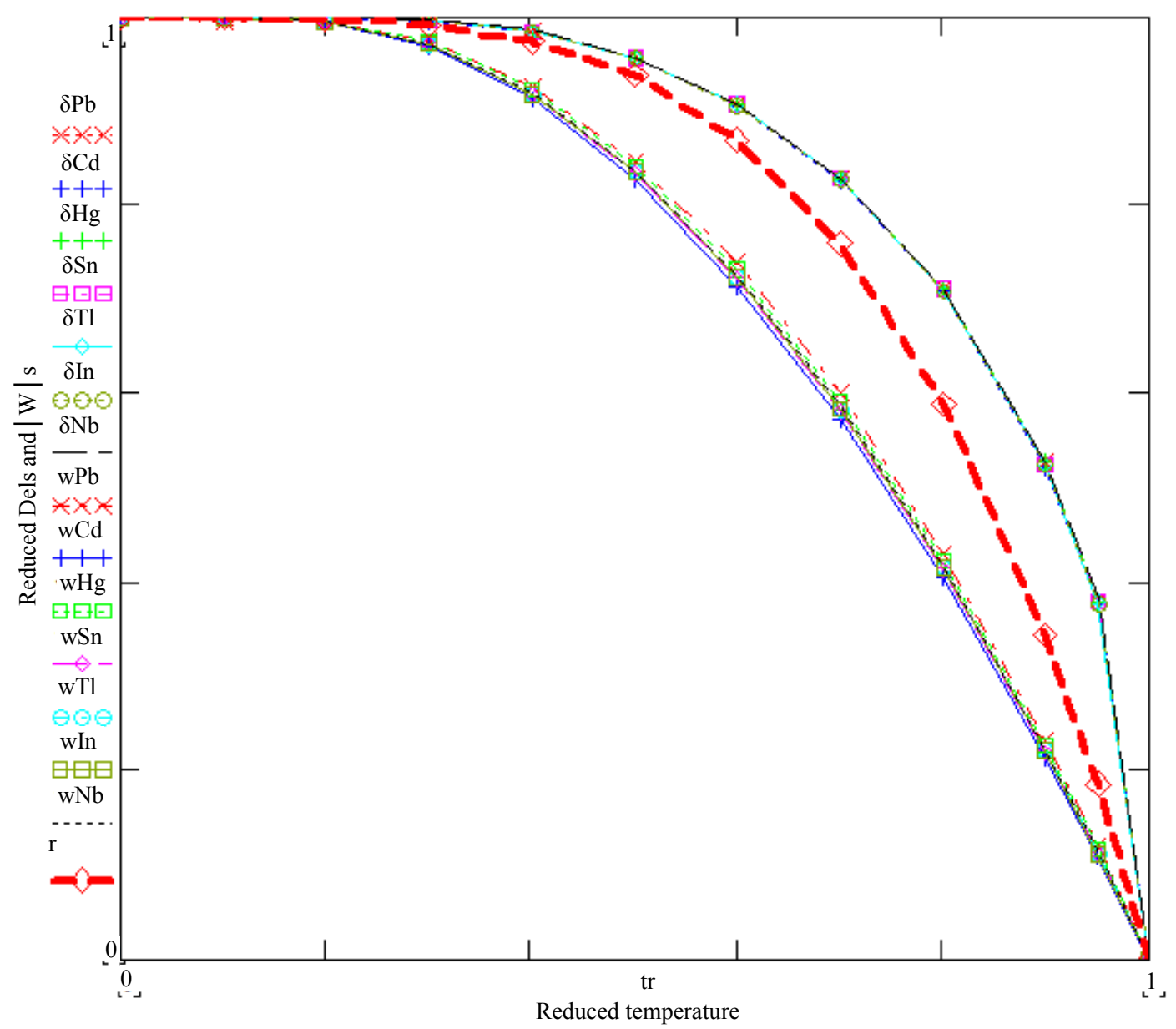

Figure 1. Plots of $\Delta(T) / \Delta_{0}$ obtained via (2), and of $|W(T)| /\left|W_{0}\right|$ obtained via (4). $\lambda$ in each case is taken to be $\lambda\left(T_{c}\right)$ as given in Table 1. The upper cluster of curves corresponds to $\Delta(T) / \Delta_{0}$, and the lower to $|W(T)| /\left|W_{0}\right|$. The thick dashed curve between the two clusters corresponds to $n_{s}=1-t^{4}$, see (9).

recall that it was pointed out by Pines [7] that $\lambda$ can be decoupled into a product of two rather disparate quantities $N(0)$ and $V$. The first of these is the density of states at the Fermi surface

$$
N(0)=\frac{3 \gamma}{2 \pi^{2} k_{B}^{2} v},
$$

where $\gamma$ is the Sommerfeld parameter, $v$ the gram-atomic volume of the element, and $V$ is the algebraic sum of the attractive interaction between electrons owing to the lattice and the repulsive Coulomb interaction. We now ask as to how $V$ might change for an SC if, starting from $T>$ $T_{c}$, its temperature is progressively lowered to $T=0$.

We recall that Cooper showed a long time ago that - and this is the basis of virtually all theories that attempt to explain superconductivity - pair-formation will take place however small the net attraction between electrons may be, causing a lowering of the energy of the system and hence superconductivity to arise. This sug- gests that for all $T>T_{c}, V$ is positive, changes sign at $T=$ $T_{c}$ and continues to (negatively) increase so as to attain its maximum value at $T=0$. Such $T$-dependence of $\lambda$ is required in addition to the $T$-variation because of the tanh term in (1) in order to cause closure of the two gaps of a binary at the higher $T_{c}$, as will be seen in the next section. There is, however, another interesting possibility: $V$ is attractive even for $T>T_{c}$ (i.e., there is no change of sign at $T=T_{c}$ ), but (negatively) keeps increasing down to $T=$ 0 . If this be so, then there must be CPs without there being superconductivity. This suggests the existence of "preformed CPs" before superconductivity sets in $[8,9]$; whether or not this feature has a bearing on the so-called pseudogaps in HTSCs seems still to be an open question.

8 ) In connection with the $T$-dependence of $\lambda$, we note that in a recent study [10] it has been shown that one can account for the empirical law that relates the critical magnetic field $H_{c}(T)$ of an elemental SC with $H_{c}(0)$ by assuming that $V$ varies linearly with temperature. 
9) Assuming that $\lambda$ in (1) varies linearly with $t$, we have $\lambda(t)_{E q 2}=\lambda\left(\Delta_{0}\right)-\alpha T_{c} t,\left(\alpha=\left[\lambda\left(\Delta_{0}\right)-\lambda\left(T_{c}\right)\right] / T_{c}\right)$.

Using the values of $\lambda s$ in Table 1, $\alpha$ is found to have the values $7.381 \times 10^{-3}, 3.819 \times 10^{-3}, 6.867 \times 10^{-3}, 7.796$ $\times 10^{-4}, 9.971 \times 10^{-4}, 2.857 \times 10^{-3}$, and $8.649 \times 10^{-4}$, for $\mathrm{Cd}, \mathrm{Pb}, \mathrm{Hg}, \mathrm{Sn}, \mathrm{In}, \mathrm{Tl}$, and $\mathrm{Nb}$, respectively.

Assuming a similar variation for the $\lambda$ in (3)

$$
\begin{aligned}
& \lambda(t)_{E q 4}=\lambda\left(\left|W_{01}\right|\right)-\alpha_{1} T_{c} t, \\
& \left(\alpha_{1}=\left[\lambda\left(\left|W_{01}\right|\right)-\lambda\left(T_{c}\right)\right] / T_{c}\right),
\end{aligned}
$$

we find that $\alpha_{1}$, for the same elements in the same order, has the following values: $7.143 \times 10^{-3}, 2.319 \times 10^{-3}, 5.47$ $\times 10^{-3}, 4.839 \times 10^{-4}, 3.519 \times 10^{-4}, 1.933 \times 10^{-3}$, and $5.388 \times 10^{-3}$.

For $0 \leq t \leq 1$, we now solve (1) for $\Delta(T) / \Delta_{0}$ using (13), and (3) for $\left|W_{01}(T)\right| /\left|W_{01}\right|$ using (14). As seen from Figure 2, the cluster of curves corresponding to the latter is more compact than the cluster for the former. This is one of our main results because of its universality unlike, strictly speaking, the alleged universality of the constancy of the gap-to- $T_{c}$ ratio.

We note in passing that the cluster for $\left|W_{01}(T)\right| /\left|W_{01}\right|$ is reproduced rather well by $n_{s}(t)=1-t^{2.6}$.

\section{Dealing with Binary SCs via BCSEs Compared with GBCSEs}

An important feature of our considerations concerned with elemental SCs above is that we have been willynilly led to the idea of $T$-dependent coupling constants. We now draw attention to the need for such an idea even for composite (i.e., non-elemental) SCs that are characterized by two $\Delta_{0} s$ but one $T_{c}$. For the sake of concreteness, let us consider the case of YBCO for which the

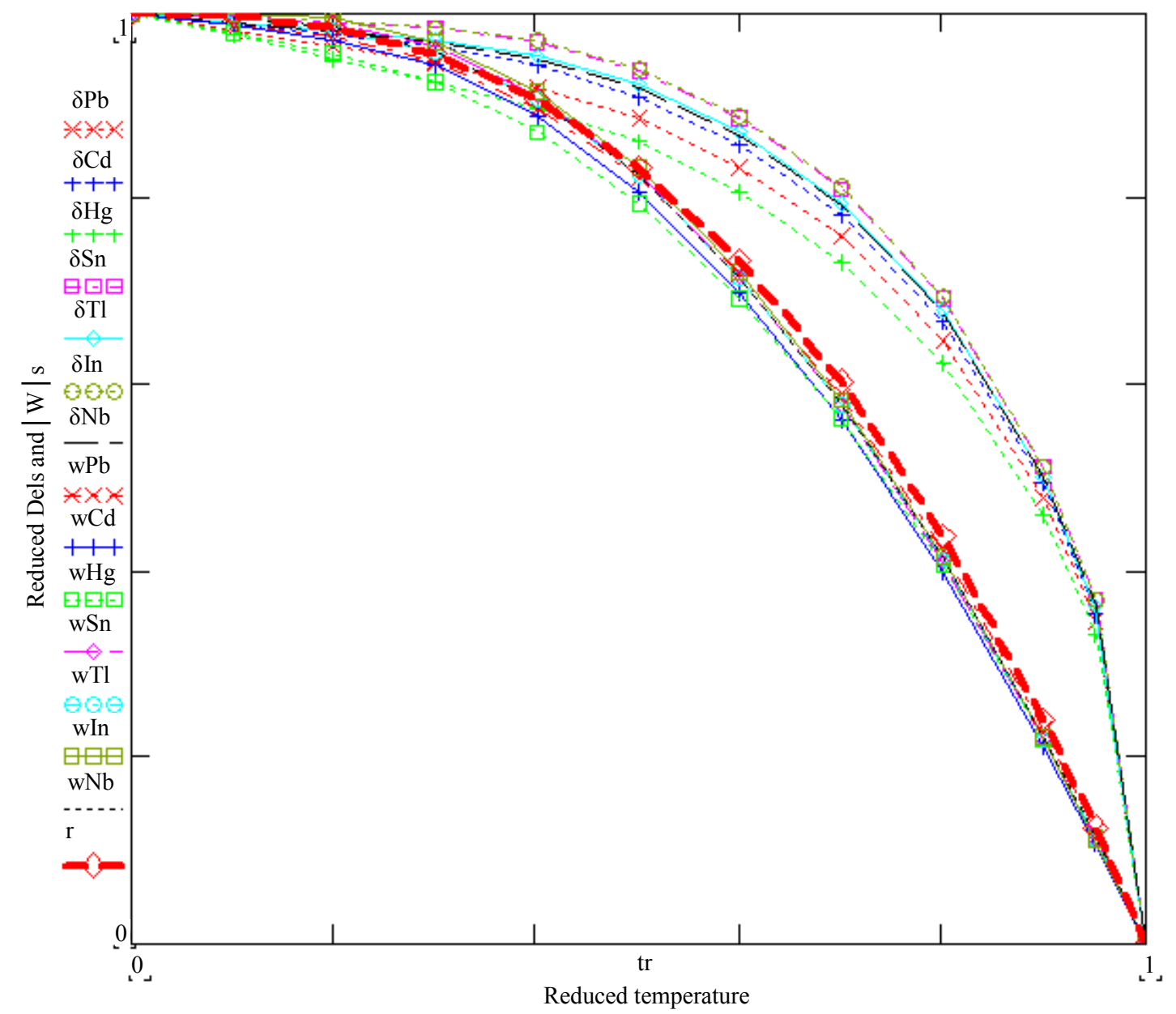

Figure 2. Plots of $\Delta(T) / \Delta_{0}$ obtained via (2) with variable $\lambda$ given in (11), and of $|W(T)| /\left|W_{0}\right|$ obtained via (4) with $\lambda$ given in (12). The upper cluster of curves corresponds to $\Delta(T) / \Delta_{0}$, and the lower to $|W(T)| /\left|W_{0}\right|$. The thick dashed curve corresponds to $n_{s}=1-t^{2.6}$. 
characteristic set of parameters $\left\{\Delta_{01}, \Delta_{02}, T_{c}, \Theta\right\}$ is given by [3].

$$
\{5.5 \mathrm{meV}, 20.0 \mathrm{meV}, 92 \mathrm{~K}, 410 \mathrm{~K}\}
$$

It was pointed out by Suhl et al. [5] that itinerancy of electrons between the $s$ - and the $d$-bands and the overlap between them can cause an SC to have two gaps via (1) and, in general, two critical temperatures via (3) within the framework of BCS theory. This happens because $\lambda$ is now given by a quadratic equation. Indeed, this is the approach that has frequently been invoked, albeit qualitatively, to deal with two-gapped SCs.

Let us therefore first apply this approach to YBCO.

The $\lambda s$ corresponding to the two gap values in (15) calculated via (10) are found to be 0.391 and 0.75 , leading to $T_{c}=36.2$ and $123 \mathrm{~K}$, respectively. On the other hand, $\lambda$ calculated with the input of $T_{c}$ from the set (15) into (2) is found to be 0.615 , which leads to the value of 14.4 $\mathrm{meV}$ for the larger gap. Note that we are working in the "BCS-Suhl" scenario and not in the "Eliashberg-Suhl" scenario. Therefore we posit that a value of $\lambda$ exceeding the minimum upper bound of 0.5 via the Bogoliubov criterion [11] is unacceptable. Furthermore, even if one ignores the difference between 0.75 and 0.615 , we need to close both the gaps at the higher $T_{c}$ i.e., $123 / 92 \mathrm{~K}$. We assert that this cannot be brought about without invoking $T$-dependence of the $\lambda s$. This will be explicitly shown elsewhere.

We note that, as has been shown [3,4], none of the $\lambda s$ invoked in the framework of GBCSEs to deal with the gap-values and the $T_{c}$ of any HTSC violates the Bogoliubov constraint mentioned above. In the context of YBCO, since it has already been shown as to how GBCSEs account for its superconducting properties, we need to make just one additional remark which is: for closure of the two gaps of YBCO one requires, again, the $\lambda s$ in the theory to be $T$-dependent.

Having willy-nilly been led to the not-so-familiar domain of $T$-dependent approach here, it seems pertinent to note that such an approach has factually been employed in diverse fields in the past by: 1) Bogoliubov, Zubarev and Tserkovnikov, as discussed in [11, p. 250]; Sheahen [12], and in Ref. [10] in the context of superconductivity; 2) by Weinberg [13], Linde [14], and Dolan and Jackiw [15] in the context of finite-temperature behavior of a class of relativistic field theories (RFTs) to address the question of restoration of a symmetry which at zero temperature is broken either dynamically or spontaneously; 3) in Ref. [16] for a model RFT; 4) for an explanation of the legions of unidentified solar emission lines in Ref. [17], and 5) in QCD to explain the masses of different quarkonium families and their de-confinement temperatures in Refs. [18,19].

\section{Conclusions}

1) We believe to have presented in detail a convincing case for regarding (3) as a viable alternative to (1).

2) The above finding suggests the use of both (3) and (4) whenever a detailed variation of the two gaps of a CS with $T$ is required.

3) While our main motivation for this note was to draw attention to the above feature, we have collaterally been led to the somewhat not-so-familiar domain of $T$-dependent Hamiltonians. This led us to draw attention to diverse fields where $T$-dependent Hamiltonians have fruitfully been employed in the past.

4) The well-known approach of Suhl et al. [5] to multi-gap superconductivity was recalled. It was pointed out that if experiment dictates that the two gaps of an SC close at the same (higher) $T_{c}$, then both Suhl et al.'s and the approach provided by GBCSEs need to invoke $T$-dependence of the interaction parameters.

5) We conclude by noting that considerations of this note find an immediate and important application in the study of the thermal conductivity $(\kappa)$ of high- $T_{c}$ SCs via the Geilikman [20] and Geilikman-Kresin [21] or, equivalently, the Bardeen, Rickayzen and Tewordt theories [22]. Specifically, we are now enabled to investigate the variation of $\kappa$ with $T$ both in the scenario where the two gaps of say, $\mathrm{MgB}_{2}$, close at the same $T_{c}$ and in the scenario in which they do not. We recall that thermal conductivity is a non-equilibrium phenomenon and that it is measured under conditions of no electric current. Since a thermal current tends to drag a small electric current with it, this current must be balanced by an equal and opposite supercurrent. For these reasons measurement of $\kappa$ requires a rather elaborate experimental set up. A pertinent question therefore is: Could the cumulative effect of the stresses caused by such a set up lift the degeneracy of the two gaps closing at the same $T_{c}$ ? A preliminary study shows that this is indeed so i.e., the experimental data on $\kappa(T)$ of $\mathrm{MgB}_{2}$ is better explained in the scenario in which its two gaps close at different temperatures. These findings will be reported elsewhere.

\section{Acknowledgements}

GPM thanks Prof. D. C. Mattis for correspondence having a bearing on this note. He thanks Ms. Amudhakumari for an interesting discussion - during EUROQUAM2010 -about the contents herein. MdeLl thanks UNAMDGAPA-PAPIIT (México) for partial support from grant IN106908.

\section{REFERENCES}

[1] J. Bardeen, L. N. Cooper and J. R. Schrieffer, "Theory of Superconductivity," Physical Review, Vol. 108, No. 5, 
1957, pp. 1175-1204. doi:10.1103/PhysRev.108.1175

[2] G. P. Malik, "On the Equivalence of the Binding Energy of a Cooper Pair and the BCS Energy Gap: A Framework for Dealing with Composite Superconductors," International Journal of Modern Physics B, Vol. 24, No. 9, 2010, pp. 1159-1172. doi:10.1142/S0217979210055408

[3] G. P. Malik, "Generalized BCS Equations: Applications," International Journal of Modern Physics B, Vol. 24, No. 19, 2010, pp. 3701-3712. doi:10.1142/S0217979210055858

[4] G. P. Malik and U. Malik, "A Study of the Thallium- and Bismuth-Based High-Temperature Superconductors in the Framework of the Generalized BCS Equations," Journal of Superconductivity and Novel Magnetism, Vol. 24, No. 1-2, 2011, pp. 255-260. doi:10.1007/s10948-010-1009-0

[5] H. Suhl, B. T. Matthias and L. R. Walker, "BardeenCooper-Schrieffer Theory of Super-Conductivity in the Case of Overlapping Bands," Physical Review Letters, Vol. 3, 1959, pp. 552-554.

doi:10.1103/PhysRevLett.3.552

[6] C. P. Poole, "Handbook of Superconductivity," Academic Press, San Diego, 2000, p. 48.

[7] D. Pines, "Superconductivity in the Periodic System," Physical Review, Vol. 109, No. 2, 1958, pp. 280-287. doi:10.1103/PhysRev.109.280

[8] T. Mamedov and M. de Llano, "Superconducting Pseudogap in a Boson-Fermion Model," Journal of the Physical Society of Japan, Vol. 79, No. 4, 2010, Article ID: 044706.

[9] T. Mamedov and M. de Llano, "Generalized Superconducting Gap in an Anisotropic BosonFermion Mixture with a Uniform Coulomb Field," Journal of the Physical Society of Japan, Vol. 80, No. 4, 2011, Article ID: 074718.

[10] G. P. Malik, "On Landau Quantization of Cooper Pairs in a Heat Bath," Physica B: Condensed Matter, Vol. 405, No. 16, 2011, pp. 3475-3481. doi:10.1016/j.physb.2010.05.026

[11] J. M. Blatt, "Theory of Superconductivity," Academic Press, New York, 1964, p. 206.

[12] T. P. Sheahan, "Effective Interaction Strength in Super- conductors," Physical Review, Vol. 149, No. 1, 1966, pp. 370-377. doi:10.1103/PhysRev.149.370

[13] S. Weinberg, "Gauge and Global Symmetries at High Temperature," Physical Review D, Vol. 9, No. 12, 1974, pp. 3357-3378. doi:10.1103/PhysRevD.9.3357

[14] A. D. Linde, "Phase Transitions in Gauge Theories and Cosmology," Reports on Progress in Physics, Vol. 42, No. 3, 1979, pp. 390-437. doi:10.1088/0034-4885/42/3/001

[15] L. Dolan and R. Jackiw, "Symmetry Behavior at Finite Temperture," Physical Review D, Vol. 9, No. 12, 1974, pp. 3320-3341. doi:10.1103/PhysRevD.9.3320

[16] G. P. Malik and L. K. Pande, "Wick-Cutkosky Model in the Large-Temperature Limit," Physical Review D, Vol. 37, No. 12, 1988, pp. 3742-3748. doi:10.1103/PhysRevD.37.3742

[17] G. P. Malik, L. K. Pande and V. S. Varma, "On Solar Emission Lines," The Astrophysical Journal, Vol. 379, 1991, pp. 788-795. doi:10.1086/170554

[18] G. P. Malik, R. K. Jha and V. S. Varma, "Mass Spectrum of the Temperature-Dependent Bethe-Salpeter Equation for Composites of Quarks with a Coulomb plus a Linear Kernel," The European Physical Journal A, Vol. 2, No. 1, 1998, pp. 105-110. doi:10.1007/s100500050096

[19] G. P. Malik, R. K. Jha and V. S. Varma, "Quarkonium Mass Spectra from the Temperature-Dependent BetheSalpeter Equation with Logarithmic and Coulomb plus Square-Root Kernels," The European Physical Journal A, Vol. 3, No. 4, 1998, pp. 373-375. doi: $10.1007 / \mathrm{s} 100500050191$

[20] B. T. Geilikman, "Thermal Conductivity of Super-Conductors," Soviet Physics, Vol. 7, 1958, pp. 721-722.

[21] B. T. Geilikman and V. Z. Kresin, "Phonon Thermal Conductivity of Superconductors," Soviet Physics Dolady, Vol. 3, No. 6, 1958, pp. 1161-1163.

[22] J. Bardeen, G. Rickayzen and L. Tewordt, "Theory of Thermal Conductivity of Superconductors," Physical Review, Vol. 113, No. 4, 1959, pp. 982-994. doi:10.1103/PhysRev.113.982 